\title{
Orientated molecular information from chiral rotational spectroscopy
}

\author{
Jörg B. Götte ${ }^{* a, b, c}$, Robert P. Cameron ${ }^{b, c}$ and Stephen M. Barnett ${ }^{c}$ \\ ${ }^{a}$ National Laboratory of Solid State Microstructures, College of Engineering and Applied \\ Sciences, Nanjing University, Nanjing 210093, China; \\ ${ }^{b}$ Max Planck Institute for the Physics of Complex Systems, 01187 Dresden, Germany; \\ ${ }^{c}$ School of Physics and Astronomy, Glasgow G12 8QQ, United Kingdom
}

\begin{abstract}
We present details on how the newly introduced technique of chiral rotational spectroscopy can be used to extract orientated information from otherwise freely rotating molecules in the gas phase. In this technique circularly polarized light is used to illuminate chiral molecules and shift their rotational levels to yield orientated chiroptical information via their rotational spectrum. This enables in particular the determination of the individual, physically relevant components of the orientated optical activity pseudotensor. Using the explicit example of (S)-propylene glycol we show how measuring the rotational spectrum of molecules in the microwave domain allows for the recording of a small set of rotational transitions from which the individual polarizability components can be determined.
\end{abstract}

Keywords: Chirality, Molecular Spectroscopy, Rotational Spectroscopy, Microwave Spectroscopy

\section{INTRODUCTION}

Many molecules differ in structure from their mirror image forms, a concept which has been termed 'chiral' by Lord Kelvin. ${ }^{1}$ In particular molecules important for life, such as amino acids and sugars, and also many active ingredients in medical drugs, are chiral. ${ }^{2}$ The mirror image forms, or enantiomers, of a chiral molecule can have rather different biological actions; a simple example is ibuprofen, as one enantiomer acts as an anti-inflammatory, whereas the other is ineffective. Another example is citalopram, which is used in antidepressant drugs; only one of the enantiomers of citalopram has the desired pharmaceutical effect, while the other is ineffective, while still causing adverse side effects. In both cases, pharmaceutical companies market racemic (50:50) mixtures and enantiopure drugs with a reduced dosage as different products. It is therefore easy to understand that analytic techniques probing the chirality of molecules are highly sought after, especially as in many respects our ability to probe mixtures and elusive forms of chirality remain incomplete. One particular route to improve our analytical capabilities is to find ways of evoking an orientated response from the molecule. This is because well-established, conventional techniques to investigate the chirality of molecules, such as circular dichroism ${ }^{2-4}$ and Raman optical activity ${ }^{3-5}$ only probe the isotropically averaged properties of the molecule. Any method able to determine orientated properties of a molecule immediately offers a wealth of new information about the molecule, which is only partially captured in the isotropic properties. At present such orientated information can only be obtained from samples in the crystalline phase ${ }^{6,7}$ which is not always feasible.

Recently we have introduced a new technique termed chiral rotational spectroscopy ${ }^{8}$, which promises to enlarge our capabilities to probe molecular chirality and to analyse molecular mixtures and subtle forms of chirality, by accessing orientated information. Chiral rotational spectroscopy builds on standard rotational spectroscopy which probes the discrete rotational energy levels of a molecule typically in the microwave domain. ${ }^{9-12}$ Standard rotational spectroscopy is unable to distinguish between enantiomers as the rotational levels of the mirror image forms of a chiral molecule are identical. However, illuminating the molecules with circularly polarized light in the visible, and therefore far-off resonance in terms of the rotational transitions, evokes an optical response from

Further author information: (Send correspondence to goette@nju.edu.cn) 
the molecules which depends on the rotational states of the molecule as the chiralities of the molecule and the light. ${ }^{13}$

Based on the ability to determine the individual components of the optical activity pseudotensor, chiral rotational spectroscopy enables a number of important sensitivities:

1. the ability to measure the enantiomeric excess of a sample of chiral molecules, while giving an incisive result about the chirality of molecules even for a racemic mixture. It is therefore not necessary to prepare a sample in which one enantiomer is prevalent, which means that chiral rotational spectroscopy does not require dissymmetric synthesis or resolution of the sample to be investigated.

2. the ability to probe the chirality of molecules that are only chiral because one or more of the constituting atoms have been substituted for different isotopes. Traditional techniques often have difficulties in recognising this subtle form of chirality referred to as isotopic chirality.

3. the ability to distinguish clearly between subtly different molecular forms, which can arise for molecules with multiple chiral centres, while retaining the analytic capabilities of resolving the individual components and their chirality.

In this paper we briefly review the theoretical background of chiral rotational spectroscopy in section 2 before working through the example of (S)-propylene glycol to explain how a set of measured rotational transition frequencies can be used to gain orientated information about a molecule, including individual components of the molecule's optical activity pseudotensor, in section 3. As a caveat it should be noted that chiral rotational spectroscopy is a technique to analyse measured rotational spectra to gain orientated information about molecules. However, in the absence of experimental data, we make use of numerically calculated chiroptical properties in order to predict required rotational spectra, such that we can explain the general method of extracting the desired information from the rotational spectrum. The paper ends with a short discussion and outlook on future work in section 4.

\section{THEORY}

The detailed theory of chiral rotational spectroscopy is presented in our recent article in Physical Review A. ${ }^{8}$ Here we summarise the key elements needed to appreciate the example in the next section. We work in the International System of Units, with $\epsilon_{0}$ the vacuum permittivity, $c$ the vacuum speed of light and $\hbar=h / 2 \pi$ the reduced Planck constant.

Consider a small, chiral molecule in the gas phase, illuminated for a short time by weak, far off-resonance circularly polarised light to which it has been adiabatically introduced. Let us assume the molecule to be in a ${ }^{1} \Sigma$ vibronic state and neglect the effects of nuclear spin and translation, for the sake of simplicity.

The illuminating light drives oscillations in the charge and current distributions of the molecule, affecting the rotation of the molecule whilst shifting its energy in an orientated, chiral manner: to leading order the energy shift is

$$
\frac{I}{\epsilon_{0} c}\left[\mathcal{A}\left(\frac{1}{2} \alpha_{X X}+\sigma k B_{X X}\right)+\mathcal{B}\left(\frac{1}{2} \alpha_{Y Y}+\sigma k B_{Y Y}\right)+\mathcal{C}\left(\frac{1}{2} \alpha_{Z Z}+\sigma k B_{Z Z}\right)\right]
$$

where $I, k$ and $\sigma$ are the intensity, wavenumber and polarisation parameter of the light, with $\sigma= \pm 1$ for leftor right-handed circular polarization; $X, Y$ and $Z$ are molecule-fixed coordinates; the constants $\mathcal{A}, \mathcal{B}$ and $\mathcal{C}$ with $\mathcal{A}+\mathcal{B}+\mathcal{C}=1$ are particular to the rotational state of the molecule; $\alpha_{X X}, \alpha_{Y Y}$ and $\alpha_{Z Z}$ are orientated polarisability tensor components, which are each identical for opposite enantiomers, ${ }^{3,4}$ and $B_{X X}, B_{Y Y}$ and $B_{Z Z}$ are orientated chiroptical response pseudotensor components, which are each of equal magnitude but opposite sign for opposite enantiomers. ${ }^{14,15}$

When the rotational spectrum of such molecules is measured, typically using microwaves, it appears distorted due to the light: the light-shifted resonance frequency for a given rotational transition and enantiomer is

$$
\Delta f_{\sigma}=\Delta f_{0}+\frac{I}{2 \pi \epsilon_{0} c \hbar}\left[\Delta \mathcal{A}\left(\frac{1}{2} \alpha_{X X}+\sigma k B_{X X}\right)+\Delta \mathcal{B}\left(\frac{1}{2} \alpha_{Y Y}+\sigma k B_{Y Y}\right)+\Delta \mathcal{C}\left(\frac{1}{2} \alpha_{Z Z}+\sigma k B_{Z Z}\right)\right]
$$


where $\Delta f_{0}$ is the unperturbed transition frequency and $\Delta \mathcal{A}, \Delta \mathcal{B}$ and $\Delta \mathcal{C}$ are differences in the values of $\mathcal{A}, \mathcal{B}$ and $\mathcal{C}$ for the particular rotational states involved.

Suppose now that this frequency $\left(\Delta f_{\sigma}\right)$ has been measured for both circular polarizations $(\sigma=1$ and $\sigma=-1)$. The centred average $\left(\delta f_{a}\right)$ of the measured frequencies $\left(\Delta f_{+1}\right.$ and $\left.\Delta f_{-1}\right)$ yields a certain combination of the orientated polarisability tensor components $\alpha_{X X}, \alpha_{Y Y}$ and $\alpha_{Z Z}$ and hence orientated, achiral information about the molecules:

$$
\delta f_{a}=\frac{\Delta f_{+1}+\Delta f_{-1}}{2}-\Delta f_{0}=\frac{I}{4 \pi \epsilon_{0} c \hbar}\left[\Delta \mathcal{A} \alpha_{X X}+\Delta \mathcal{B} \alpha_{Y Y}+\Delta \mathcal{C} \alpha_{Z Z}\right] .
$$

This is the achiral component of the light-induced shift in the resonance frequency. The difference $\left(\delta f_{c}\right)$ of the measured frequencies $\left(\Delta f_{+1}\right.$ and $\left.\Delta f_{-1}\right)$ yields a certain combination of the orientated chiroptical response pseudotensor components $B_{X X}, B_{Y Y}$ and $B_{Z Z}$ and hence orientated, chiral information about the molecules:

$$
\delta f_{c}=\Delta f_{+1}-\Delta f_{-1}=\frac{I k}{\pi \epsilon_{0} c \hbar}\left[\Delta \mathcal{A} B_{X X}+\Delta \mathcal{B} B_{Y Y}+\Delta \mathcal{C} B_{Z Z}\right] .
$$

The is the 'chiral splitting' in the light-shifted resonance frequency: the separation between the resonance frequencies measured for opposite circular polarizations. Similarly for a given circular polarization and opposite enantiomers. The splitting can be seen in a single spectrum if both enantiomers are present, which is how the chirality of molecules in racemic mixtures can be probed using our technique. ${ }^{8}$

In the next section we show how $\alpha_{X X}, \alpha_{Y Y}, \alpha_{Z Z}, B_{X X}, B_{Y Y}$ and $B_{Z Z}$ can be determined individually from $\delta f_{a}$ and $\delta f_{c}$, assuming the isotropic sums $\bar{\alpha}=\left(\alpha_{X X}+\alpha_{Y Y}+\alpha_{Z Z}\right) / 3$ and $\bar{B}=\left(B_{X X}+B_{Y Y}+B_{Z Z}\right) / 3$ to be known.

\section{EXAMPLE}

In order to determine the individual components $\alpha_{X X}, \alpha_{Y Y}$ and $\alpha_{Z Z}$ of the polarisability tensor and the individual components $B_{X X}, B_{Y Y}$ and $B_{Z Z}$ of the chiroptical response pseudotensor we need knowledge of the isotropic sums $\bar{\alpha}$ and $\bar{B}$ as well as the values of $\delta f_{a}$ and $\delta f_{c}$ for two different rotational transitions. We label rotational transitions as $J_{\tau^{\prime}, m^{\prime}}^{\prime} \leftarrow J_{\tau, m}$ with $J \in\{0,1, \ldots\}$ determining the magnitude of the rotor angular momentum, $\tau \in\{0, \ldots, \pm J\}$ labelling the rotor energy and $m \in\{0, \ldots, \pm J\}$ determining the $z$ component of the rotor angular momentum (quantised parallel to the direction of propagation of the light) ${ }^{9,12,16}$ for a particular rotational state.

Let us consider the transitions $2_{-1,0} \leftarrow 1_{-1,0}$ and $1_{-1,0} \leftarrow 0_{0,0}$, for example. We find

$$
\begin{aligned}
& 2_{-1,0} \leftarrow 1_{-1,0}: \quad \Delta \mathcal{A}=-\frac{3}{35}, \Delta \mathcal{B}=\frac{4}{35}, \quad \Delta \mathcal{C}=-\frac{1}{35} ; \\
& 1_{-1,0} \leftarrow 0_{0,0}: \quad \Delta \mathcal{A}=\frac{2}{15}, \quad \Delta \mathcal{B}=-\frac{1}{15}, \quad \Delta \mathcal{C}=-\frac{1}{15} .
\end{aligned}
$$

The situation is illustrated in Fig. 1 for a particular molecule.

A set of linear equations for $\alpha_{X X}, \alpha_{Y Y}$ and $\alpha_{Z Z}$ can now be constructed:

$$
\begin{aligned}
\delta f_{a}\left(2_{-1,0} \leftarrow 1_{-1,0}\right) & =\frac{I}{4 \pi \epsilon_{0} c \hbar}\left[-\frac{3}{35} \alpha_{X X}+\frac{4}{35}, \alpha_{Y Y}-\frac{1}{35} \alpha_{Z Z}\right], \\
\delta f_{a}\left(1_{-1,0} \leftarrow 0_{0,0}\right) & =\frac{I}{4 \pi \epsilon_{0} c \hbar}\left[\frac{2}{15} \alpha_{X X}-\frac{1}{15}, \alpha_{Y Y}-\frac{1}{15} \alpha_{Z Z}\right]
\end{aligned}
$$

together with $\bar{\alpha}=\frac{1}{3} \alpha_{X X}+\alpha_{Y Y}+\alpha_{Z Z}$ is solved by

$$
\begin{aligned}
\alpha_{X X} & =\bar{\alpha}+\frac{4 \pi \epsilon_{0} c \hbar}{I} 4 \delta f_{a}\left(1_{-1,0} \leftarrow 0_{0,0}\right), \\
\alpha_{Y Y} & =\bar{\alpha}+\frac{4 \pi \epsilon_{0} c \hbar}{I}\left[14 \delta f_{a}\left(2_{-1,0} \leftarrow 1_{-1,0}\right)+4 \delta f_{a}\left(1_{-1,0} \leftarrow 0_{0,0}\right)\right] \\
\alpha_{Z Z} & =\bar{\alpha}-\frac{4 \pi \epsilon_{0} c \hbar}{I} 14\left[\delta f_{a}\left(2_{-1,0} \leftarrow 1_{-1,0}\right)+\delta f_{a}\left(1_{-1,0} \leftarrow 0_{0,0}\right)\right] .
\end{aligned}
$$



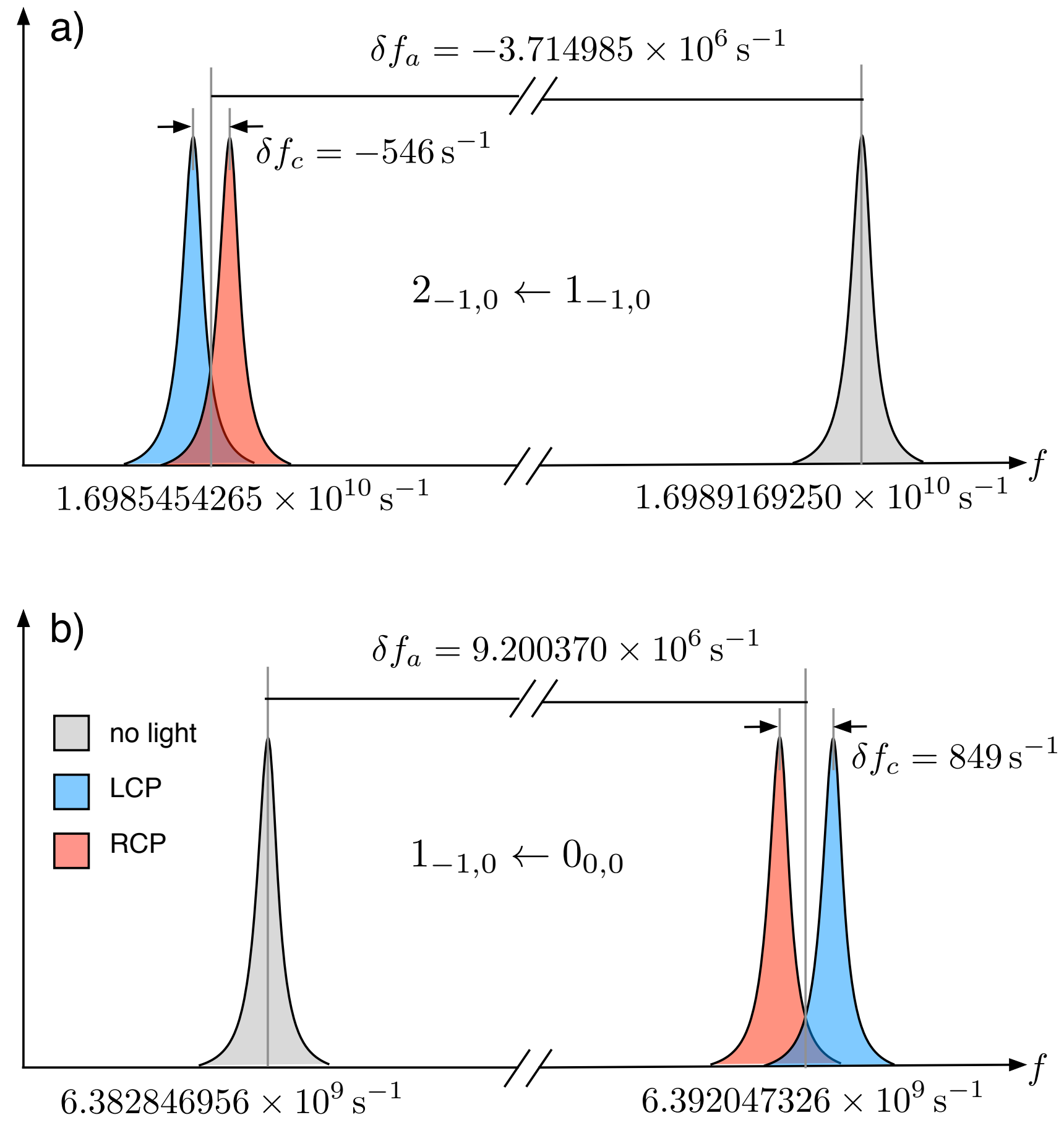

Figure 1. Rotational Spectra - Rotational lines for an enantiopure sample of the lowest energy conformer of (S)propylene glycol for the rotational transition $2_{-1,0} \leftarrow 1_{-1,0}$ in (a) and $1_{-1,0} \leftarrow 0_{0,0}$ in (b). The spectra show the lines without illumination (grey) as well as the difference for illumination with left-handed (blue) and right-handed (red) circular polarization. The graphs show the achiral frequency shift $\delta f_{a}$ and the chiral splitting $\delta f_{c}$ for illumination with an intensity of $I=2 \times 10^{12} \mathrm{~kg} \mathrm{~s}^{-3}=2 \times 10^{8} \mathrm{~W}(\mathrm{~cm})^{-2}$ at a wavelength of $\lambda=2 \pi / k=532 \mathrm{~nm}$. 
A similar set of linear equations for $B_{X X}, B_{Y Y}$ and $B_{Z Z}$ can also be constructed:

$$
\begin{aligned}
\delta f_{c}\left(2_{-1,0} \leftarrow 1_{-1,0}\right) & =\frac{I k}{\pi \epsilon_{0} c \hbar}\left[-\frac{3}{35} B_{X X}+\frac{4}{35}, B_{Y Y}-\frac{1}{35} B_{Z Z}\right], \\
\delta f_{c}\left(1_{-1,0} \leftarrow 0_{0,0}\right) & =\frac{I k}{\pi \epsilon_{0} c \hbar}\left[\frac{2}{15} B_{X X}-\frac{1}{15}, B_{Y Y}-\frac{1}{15} B_{Z Z}\right]
\end{aligned}
$$

together with $\bar{B}=\frac{1}{3}\left(B_{X X}+B_{Y Y}+B_{Z Z}\right)$ is solved by

$$
\begin{aligned}
B_{X X} & =\bar{B}+\frac{\pi \epsilon_{0} c \hbar}{I k} 4 \delta f_{a}\left(1_{-1,0} \leftarrow 0_{0,0}\right), \\
B_{Y Y} & =\bar{B}+\frac{\pi \epsilon_{0} c \hbar}{I k}\left[14 \delta f_{a}\left(2_{-1,0} \leftarrow 1_{-1,0}\right)+4 \delta f_{a}\left(1_{-1,0} \leftarrow 0_{0,0}\right)\right] \\
B_{Z Z} & =\bar{B}-\frac{\pi \epsilon_{0} c \hbar}{I k} 14\left[\delta f_{a}\left(2_{-1,0} \leftarrow 1_{-1,0}\right)+\delta f_{a}\left(1_{-1,0} \leftarrow 0_{0,0}\right)\right] .
\end{aligned}
$$

This shows how measurements of light-shifted rotational transition frequencies can be used to extract orientated information about a molecule in the form of the individual components $\alpha_{X X}, \alpha_{Y Y}$ and $\alpha_{Z Z}$ of the polarisability tensor and the individual components $B_{X X}, B_{Y Y}$ and $B_{Z Z}$ of the chiroptical response pseudotensor.

However, care has to be taken so select the rotational transitions as some choices may result in linear dependent equations and hence do not give a solution for the individual components. Also in practice, reducing the number of measurements will improve the overall performance of our technique when implemented.

\section{DISCUSSION AND OUTLOOK}

We have shown how the measurement of a couple of rotational transition frequencies, shifted by the illumination with left and right circular polarized light, can be used to determine orientated information about a molecule which is otherwise accessible only for molecules in the crystalline phase. In particular our technique is able to determine the individual components of the orientated polarisability tensor and chiroptical response pseudotensor, both of which are highly sought after, as they reveal a wealth of information about the structure, chirality and optical response of the molecule which is only partially encoded in their isotropic sum.

Access to these individual components enables a set of new sensitivities for our technique, as the ability to determine the chiral character of a sample even if it is a racemic mixture, as well as the sensitivity to isotopic and other subtle forms of chirality.

In the future we will develop our technique, while continuing to look into the properties of orientated information, in particular in connection with the emergence of chirality from an achiral molecule by a small shift in the molecules mass distribution as occurs in an isotopic substitution.

\section{ACKNOWLEDGMENTS}

This work was supported by the Engineering and Physical Sciences Research Council grants EP/M004694/1, EP/101245/1 and EP/M01326X/1, the Royal Society Research Professorship RP150122 and the alumnus programme of the Newton International Fellowship from the Royal Society as well as the Max Planck Institute for the Physics of Complex Systems.

\section{REFERENCES}

[1] Kelvin, L., [The Molecular Tactics of a Crystal], Clarendon Press, Oxford (1894).

[2] Lough, J. and Wainer, I. W., [Chirality in the Natural \& Applied Sciences], Blackwell Publishing / CRC Press (2002).

[3] Barron, L. D., [Molecular light scattering and optical activity], Cambridge University Press, Cambridge, 2nd edition ed. (2004). 
[4] Craig, D. P. and Thirunamachandran, T., [Molecular quantum electrodynamics], An, Dover, New York, NY (1998).

[5] Barron, L. D., Bogaard, M. P., and Buckingham, A. D., "Differential Raman Scattering of Right and Left Circularly Polarized Light by Asymmetric Molecules," Nature 241, 113-114 (Jan. 1973).

[6] Kaminsky, W., "Experimental and phenomenological aspects of circular birefringence and related properties in transparent crystals," Reports on Progress in Physics 63, 1575-1640 (Oct. 2000).

[7] Kahr, B. and Arteaga, O., "Arago's Best Paper," ChemPhysChem 13, 79-88 (Nov. 2011).

[8] Cameron, R. P., Götte, J. B., and Barnett, S. M., "Chiral rotational spectroscopy," Physical Review A 94, 032505-24 (Sept. 2016).

[9] Townes, C. H. and Schalow, A. L., [Microwave Spectroscopy], Dover Publications, Mineola (1975).

[10] Ramsey, N. F., [Molecular beams], International series of monographs on physics, Clarendon Press, Oxford (1956).

[11] Brown, J. M. and Carrington, A., [Rotational Spectroscopy of Diatomic Molecules], Cambridge University Press, Cambridge (2003).

[12] Bunker, P. R. and Jensen, P., [Fundamentals of Molecular Symmetry], IOP Publishing, Bristol (2005).

[13] Cameron, R. P., Götte, J. B., Barnett, S. M., and Yao, A. M., "Chirality and the angular momentum of light," Philosophical Transactions of the Royal Society A: Mathematical, Physical and Engineering Sciences 375, 20150433-17 (Jan. 2017).

[14] Buckingham, A. D. and Dunn, M. B., "Optical activity of oriented molecules," Journal of the Chemical Society A: Inorganic, Physical, Theoretical (0), 1988-4 (1971).

[15] Autschbach, J., "Time-Dependent Density Functional Theory for Calculating Origin-Independent Optical Rotation and Rotatory Strength Tensors," ChemPhysChem 12, 3224-3235 (Sept. 2011).

[16] Wang, S. C., "On the asymmetrical top in quantum mechanics," Physical Review 34, 243-252 (July 1929). 